\title{
AVALIAÇÃO DO PERFIL DE ÁCIDOS GRAXOS DE QUEIJO TIPO CHEVROTIN SIMBIOTICO
}

\section{Evaluation of the fatty acid profile of symbiotic chevrotin cheese}

\author{
Fabiana Augusta Santiago Beltrao ${ }^{\prime *}$, Carla Verônica Rodarte de Moura ${ }^{2}$, \\ Martha Suely Madruga ${ }^{1}$, Annie Elisabeth Beltrão de Andrade ${ }^{l}$
}

\begin{abstract}
RESUMO
Alimentos com bactérias probióticas ou prebióticos estão incluídos na categoria de alimentos contendo compostos bioativos que tem despertado interesse devido ao efeito benéfico para a saúde. O objetivo deste estudo foi avaliar o efeito da produção de queijos tipo "chevrotin" de leite caprino, bovino e misto com a utilização de um prebiótico (inulina), associados à utilização de um probiótico, a bactéria Bifidobacterium lactis. Foram utilizados nove queijos, alocados em um delineamento inteiramente casualizado, com fatorial ( $3 \times 3)$, em três tipos de leite e três níveis de inulina $(0 \%, 2,5 \%$ e 5,0\%). De acordo com os resultados, os percentuais de ácidos graxos saturados (AGS), a concentração dos ácidos monoinsaturados (MUFA) e a concentração dos ácidos polinsaturados (PUFA) aumentaram linearmente $(\mathrm{p}<0,01)$. Foram identificados trinta ácidos graxos, dentre estes 17 saturados, nove monoinsaturados e quatro poliinsaturados. A composição dos ácidos graxos nos diferentes tipos de queijos apresentou maior teor de ácidos graxos monoinsaturados e poliinsaturados nos queijos caprinos e misto e maior teor de ácidos graxos saturados nos queijos com leite bovino.
\end{abstract}

Palavras-chave: novos produtos; processamento de queijo; inulina.

\begin{abstract}
Foods with probiotic or prebiotic bacteria are included in the category of foods containing bioactive compounds that have aroused interest because of the

1 Universidade Federal da Paraíba (UFPB), Cidade Universitária, s/n, Castelo Branco III, 58051-900, João Pessoa, PB, Brasil. Email: fasb.15@hotmail.com

2 Universidade Federal do Piauí (UFPI), Teresina, PI, Brasil.

* Autor para correspondência.
\end{abstract}


beneficial effect on health. The objective of this study was to evaluate the effect of chevrotin cheeses from caprine, bovine and mixed milk with the use of a prebiotic (inulin), associated to the use of a probiotic, Bifidobacterium lactis. Nine cheeses were allocated in a completely randomized design with factorial (3x3) in three types of milk and three levels of inulin $(0.0 \%, 2,5 \%$ and 5,0\%). Fatty acid analysis was performed. According to the results, the percentage of saturated fatty acids (AGS) increased linearly $(p<0.01)$ the concentration of the monounsaturated acids (MUFA) and the concentration of the polyunsaturated acids (PUFA). Thirty fatty acids were identified, including 17 saturated, 9 monounsaturated and 4 polyunsaturated. The composition of fatty acids in the different types of cheeses presented a higher content of monounsaturated and polyunsaturated fatty acids in goat and mixed cheeses and higher content of saturated fatty acids in cheeses with bovine milk.

Keywords: new product; processing cheese; inulin.

\section{INTRODUÇÃO}

A aplicação de alegações de saúde na comercialização de produtos alimentícios teve início na década de 1960. Desde então, a substituição de componentes não saudáveis tais como sal, açúcar ou gordura, por componentes mais saudáveis como vitaminas, antioxidantes, fibras e bactérias láticas probióticas tem sido realizada nos alimentos (ZIEMER; GIBSON, 1998).

$\mathrm{Na}$ categoria de alimentos funcionais encontram-se os alimentos com bactérias probióticas e substratos específicos para essas bactérias consideradas benéficas, os prebióticos. Varias espécies de Bifidobacterium spp. têm sido empregadas como probióticas (ZIEMER; GIBSON, 1998). A utilização de substâncias como inulina, que é uma oligofrutose tem sido adicionadas aos alimentos devido às suas funções prebióticas (ROBERFROID, 1999; ZIEMER; GIBSON, 1998).

A Resolução RDC nº 2/2002 define probióticos como microrganismos vivos capazes de melhorar o equilíbrio microbiano intestinal produzindo efeitos benéficos à saúde do consumidor (ANVISA, 2002). Várias funções benéficas dos probióticos são descritas como: restabelecimento da microbiota intestinal balanceada; resistência à colonização e/ ou prevenção de diarreia; redução sistêmica do colesterol sérico, redução de enzimas fecais e mutagênicos potenciais que possam induzir tumores; metabolismo da lactose e redução da intolerância à lactose; melhora na resposta do sistema imune; melhor absorção de cálcio e síntese de vitaminas e pré-digestão (YUHARA, et al. 2014).

Os alimentos com propriedades funcionais são todos os alimentos ou bebidas que consumidas diariamente, possuem ingredientes fisiologicamente saudáveis e podem trazer benefícios específicos aos seres humanos e, nesse caso, os prebióticos e probióticos podem ser os principais ingredientes desses alimentos (CANDIDO; CAMPOS, 2005). Bactérias do gênero Bifidobaterium são mais frequentemente empregadas como suplemento probiótico para alimentos, uma vez que elas estão sendo isoladas de todas as porções do trato gastrointestinal do humano saudável (BIELECKA et al., 2002).

A cromatografia é um conjunto de métodos de separação com características básicas comuns. As separações envolvem o transporte dos componentes de uma mistura líquida ou gasosa através de uma coluna. A coluna contém um material, em forma de fase estacionária, que consiste em um agente sólido adsorvente ou um agente líquido distribuidor. 
Em virtude do retardamento seletivo exercido pela fase estacionária, os componentes da mistura se movimentam através da coluna a diferentes velocidades efetivas e a migração diferencial tende a segregar os componentes em zonas ou bandas separadas (CARVALHO, 2000).

A CG é a técnica que mais tem sido aplicada ao estudo dos ácidos graxos, devido principalmente ao desenvolvimento de fases estacionárias que possibilitam a separação dos diversos isômeros posicionais e geométricos existentes na mistura (SIMIONATO, 2008). Segundo Tvrzická et al. (2002) a cromatografia gasosa permite a identificação dos componentes da amostra através da comparação dos tempos de retenção dos compostos, com aqueles obtidos através da injeção de padrões contendo as substâncias a serem analisadas. No entanto, este procedimento não é conclusivo, pois componentes diferentes podem ter o mesmo tempo de retenção (MILINSK et al., 2008). A quantificação dos lipídios nos alimentos é realizada, tradicionalmente, por extração com solventes orgânicos (éter etílico, éter de petróleo, clorofórmio e metanol) e determinação gravimétrica (COSTA, 2011).

Objetivou-se com este trabalho desenvolver um queijo tipo "chevrotin" simbiótico, a partir de leite bovino, caprino e misto com adição do prebiótico (inulina) e do probiótico (Bifidobacterium lactis) e verificar o perfil de ácidos graxos dos produtos através da cromatografia.

\section{MATERIAL E METODOS}

Para a fabricação do queijo tipo chevrotin foram utilizados três tipos de leite: bovino, caprino e misto (caprino e bovino). $\mathrm{Na}$ produção dos queijos foi utilizado um prebiótico (inulina), com diferentes níveis de concentração, associados à utilização de um probiótico, o Bifidobacterium lactis. Foram fabricados nove queijos alocados em um delineamento inteiramente casualizado, com fatorial $(3 \times 3 \times 3)$, em três períodos de 15 dias de armazenamento e três tipos de leite e três níveis de inulina $(0 \%, 2,5 \%$ e $5,0 \%)$.

O leite destinado a fabricação de queijos foi obtido de animais sadios, limpo, com aroma e sabor normais, não apresentando nenhum cheiro ou sabor estranhos e acidez baixa. Para os três tipos de leite o processamento foi o mesmo. A acidez dos leites estava entre $16-20^{\circ} \mathrm{D}$. O leite foi filtrado com peneira de nylon para se evitar impurezas.

Foram utilizados 10 litros de leite para cada fabricação sendo: 10 litros de leite de cabra (tratamento 1), 10 litros de leite de vaca (tratamento 2) e 5 litros de leite de cabra + 5 litros de leite de vaca (tratamento 3). Para essas quantidades de leite foram usados $20 \mathrm{~mL}$ de fermento lácteo, $7 \mathrm{~mL}$ de coalho químico e $4 \mathrm{~mL}$ de cloreto de cálcio.

Os leites foram pasteurizados com pasteurização lenta $\left(65^{\circ} \mathrm{C}\right.$ por 30 minutos) (ORDONEZ, 2005). Após a pasteurização os leites foram resfriados à temperatura de $35^{\circ} \mathrm{C}$, que é temperatura ótima para o crescimento de microrganismos termófilios.

Foram adicionados ao leite o coalho e a cultura láctea (Bifidobacterium lactis) (adquirido na Christian Hansen - USA) ficando em repouso por 40 minutos, a temperatura ambiente, para ocorrer à coagulação. Em seguida foi realizado lentamente o corte da coalhada, com liras, no sentido horizontal e vertical, de forma de obter grãos pequenos (tamanho do grão de milho, ou seja, $1 \mathrm{~cm}$ de aresta). A mexedura única ocorreu lentamente por cerca de 2 minutos, o dessoramento ocorreu em pano murim e em seguida foi adicionado a inulina $(0 \%, 2,5 \%$ ou $5 \%)$ adquiridos no Morelia (Sesores y Equipos de Processo, AS de Cv-México).

A maturação foi realizada em câmara fria $\left(10{ }^{\circ} \mathrm{C}\right.$ e $85 \%$ de umidade) por um período de 30 dias. Os produtos foram embalados 
em condições assépticas com papel alumínio, rotulados e armazenados sob refrigeração $\left(10{ }^{\circ} \mathrm{C}\right)$ por um período de 60 dias.

\section{Análise dos ácidos graxos}

Foram analisadas 12 amostras de leite e queijo, compostas de três tratamentos e três períodos experimentais $(3 \times 3 \times 3)$. As amostras foram homogeneizadas com bastão de vidro e retiradas alíquotas de $100 \mathrm{~mL}$ de leite e estas amostras foram obtidas alíquotas de $2 \mathrm{~g}$ para os processos de extração, saponificação e esterificação (FOLCH et al., 1957). Em seguida foi realizada a metilação de acordo com metodologia de Hartman e Lago (1973). Para determinação quantitativa dos ácidos graxos utilizou-se um cromatógrafo a gás (CG VARIAN CP - 3380), com detector de ionização de chama (FID), coluna capilar (SP- 2560) de 100 metros x 0,25 mm (d.i.) $\mathrm{x} 0,20 \mu \mathrm{m}$, nitrogênio como gás de arraste (30 mL/ min), hidrogênio (10 $\mathrm{mL} / \mathrm{min})$ e ar sintético $(300 \mathrm{~mL} / \mathrm{min})$. O tempo total de corrida foi de 63 minutos, cuja temperatura da coluna iniciou a $70{ }^{\circ} \mathrm{C}$ permanecendo por 4 min., posteriormente a temperatura da coluna foi elevada a $8{ }^{\circ} \mathrm{C} / \mathrm{min}$ para $110^{\circ} \mathrm{C}$. Em seguida elevou-se a temperatura para $170{ }^{\circ} \mathrm{C}$ a uma taxa de $5{ }^{\circ} \mathrm{C} / \mathrm{min}$ permanecendo por 10 min., posteriormente a temperatura foi aumentada, a $4{ }^{\circ} \mathrm{C} / \mathrm{min}$, para a temperatura final de $240{ }^{\circ} \mathrm{C}$, que foi mantida por 14,5 minutos. Utilizou-se amostra padrão para ácidos graxos de leite de cabra fornecida pela SUPELCO (37 Component Fame Mix, numa concentração de $10000 \mu \mathrm{g} / \mathrm{mL}$ ).

Os tempos de retenção e as percentagens dos ácidos graxos foram obtidos a partir do software SYSTEM CONTROL VARIAN STAR e expressos em percentual de área. Os resultados das análises de ácidos graxos foram compilados em planilhas eletrônicas sendo submetidas à análise de variância e regressão. As análises estatísticas foram rea- lizadas com auxílio do software SAS, versão 6.2 (SAS, 2014).

Inicialmente, os lipídeos foram extraídos das amostras de queijo segundo método descrito por Folch et al. (1957). Do extrato lipídico, tomou-se uma alíquota de $5 \mathrm{~mL}$ para a execução do processo de preparação dos ésteres metílicos, seguindo-se o método descrito por Hartman e Lago (1973). As amostras transmetiladas foram analisadas em cromatógrafo a gás injetando-se uma alíquota de $1 \mu \mathrm{L}$ do extrato esterificado e a identificação dos ácidos graxos foi feita pela comparação dos tempos de retenção e as percentagens dos ácidos graxos foram calculadas mediante o software Peaksimple (SRI Instruments - USA). Os ácidos graxos foram quantificados por normalização das áreas dos ésteres metílicos e os resultados foram expressos em percentual de área (\%).

\section{RESULTADOS E DISCUSSÃO}

O leite é composto por mais de $100 \mathrm{mil}$ tipos diferentes de moléculas, contém de 3\% a 5\% de lipídios totais, representados principalmente por triacilgliceróis encontrados nos glóbulos de gordura (PELLEGRINI et al., 2012). O ácido linoléico conjugado (CLA) consiste em um grupo de ácidos graxos encontrado no leite de animais ruminantes, como bovinos e caprinos (PRANDINI et al., 2007). O perfil de ácidos graxos das amostras dos leites, bovino, caprino e misto, bem como dos queijos conforme mostra a Tabela 1 .

Para os três tipos de leite, os ácidos graxos C:14, C:16 e C:18 apresentaram maior porcentagem, sendo significativamente superior aos demais ácidos graxos $(\mathrm{p} \leq 0,01)$. Já para os ácidos graxos monoinsaturados verificou-se que $C: 14: 1, \omega-5$ cis, $C: 16: 1$, $\omega-7$ cis, C:18:1, $\omega-9$ trans e $C: 18: 1, \omega-11$ cis apresentaram maior porcentagem, principalmente no leite bovino, sendo significativamente superior aos demais ácidos graxos $(\mathrm{p} \leq 0,01)$. 
Tabela 1 - Ácidos graxos presentes nos leites de cabra, bovino e misto

\begin{tabular}{|c|c|c|c|c|}
\hline \multicolumn{2}{|l|}{ ÁCIDOS GRAXOS } & \multirow[t]{2}{*}{$\mathrm{LC}(\%)$} & \multirow[t]{2}{*}{$\operatorname{LM}(\%)$} & \multirow[t]{2}{*}{$\mathrm{LV}(\%)$} \\
\hline Fórmula & Nome & & & \\
\hline $\mathrm{C} 4: 0$ & Butírico & $0,08^{\mathrm{c}}$ & $1,98^{\mathrm{c}}$ & $0,10^{\mathrm{c}}$ \\
\hline C6:0 & Capróico & $0,77^{\mathrm{b}}$ & $0,88^{\mathrm{ab}}$ & $0,00^{\mathrm{d}}$ \\
\hline C8:0 & Caprílico & $0,04^{\mathrm{e}}$ & $0,56^{\mathrm{b}}$ & $0,07^{\mathrm{e}}$ \\
\hline $\mathrm{C} 10: 0$ & Cáprico & $1,33^{\mathrm{g}}$ & $3,15^{\mathrm{c}}$ & $1,66^{\mathrm{g}}$ \\
\hline C11:0 & Undecanóico & $0,07^{\mathrm{c}}$ & $0,11 b^{c}$ & $0,05^{\mathrm{c}}$ \\
\hline C12:0 & Láurico & $2,15^{\mathrm{b}}$ & $2,57^{b}$ & $2,51^{\mathrm{d}}$ \\
\hline C14:0 & Mirístico & $9,36^{\mathrm{c}}$ & $9,29^{\mathrm{c}}$ & $10,30^{\mathrm{b}}$ \\
\hline C15:0 & Pentadecanóico & $1,05^{\mathrm{b}}$ & $0,91^{\mathrm{c}}$ & $1,06^{\mathrm{b}}$ \\
\hline C16:0 & Palmítico & $27,65^{\mathrm{c}}$ & $27,73^{\mathrm{c}}$ & $29,78^{\mathrm{a}}$ \\
\hline $\mathrm{C} 17: 0$ & Margárico & $0,66^{\mathrm{c}}$ & $0,66^{\mathrm{c}}$ & $0,72^{\mathrm{b}}$ \\
\hline $\mathrm{C} 18: 0$ & Esteárico & $17,46^{\mathrm{a}}$ & $16,18^{\mathrm{ab}}$ & $16,54^{\mathrm{a}}$ \\
\hline C19:0 & Nonadecanóico & $0,30^{\mathrm{a}}$ & $0,23^{\mathrm{b}}$ & $0,28^{\mathrm{b}}$ \\
\hline $\mathrm{C} 20: 0$ & Araquídico & $0,26^{\mathrm{bc}}$ & $0,23^{\mathrm{c}}$ & $0,22^{\mathrm{bc}}$ \\
\hline $\mathrm{C} 21: 0$ & Heneicosanoico & $0,37^{\mathrm{b}}$ & $0,28^{\mathrm{bc}}$ & $0,24^{\mathrm{bc}}$ \\
\hline $\mathrm{C} 22: 0$ & Behênico & $0,15^{\mathrm{b}}$ & $0,07^{\mathrm{bcd}}$ & $0,14^{\mathrm{bc}}$ \\
\hline $\mathrm{C} 23: 0$ & Tricosanóico & $2,49^{\mathrm{b}}$ & $1,50^{\mathrm{e}}$ & $0,91^{\mathrm{e}}$ \\
\hline $\mathrm{C} 24: 0$ & Lignocérico & $0,19^{\mathrm{ab}}$ & $0,24^{\mathrm{ab}}$ & $0,00^{\mathrm{d}}$ \\
\hline \multicolumn{5}{|c|}{ MONOINSATURADOS } \\
\hline $\mathrm{C} 14: 1, \omega-5$ cis & Fisetérico & $1,11^{\mathrm{a}}$ & $0,43^{\text {cd }}$ & $0,84^{\mathrm{ab}}$ \\
\hline $\mathrm{C} 15: 1, \omega-5$ cis & cis-Pentadecanoico & $0,32^{\mathrm{a}}$ & $0,31^{\mathrm{bc}}$ & $0,31^{\mathrm{bc}}$ \\
\hline $\mathrm{C} 16: 1, \omega-7 \mathrm{cis}$ & Palmitoléico & $1,07^{\mathrm{ab}}$ & $0,98^{\mathrm{bc}}$ & $1,45^{\mathrm{a}}$ \\
\hline $\mathrm{C} 17: 1, \omega-7 \mathrm{cis}$ & cis-heptadecanoico & $0,19^{\mathrm{b}}$ & $0,25^{\mathrm{a}}$ & $0,27^{\mathrm{a}}$ \\
\hline $\mathrm{C} 18: 1, \omega-9$ trans & trans Elaídico & $26,50^{\mathrm{ab}}$ & $25,12^{\mathrm{bc}}$ & $25,82^{\mathrm{bc}}$ \\
\hline $\mathrm{C} 18: 1, \omega-11 \mathrm{cis}$ & cis-vacênico & $3,17^{\mathrm{ab}}$ & $2,76^{\mathrm{cd}}$ & $3,50^{\mathrm{a}}$ \\
\hline $\mathrm{C} 20: 1, \omega-9$ cis & Gadoleico & $0,12^{\mathrm{c}}$ & $0,32^{\mathrm{a}}$ & $0,20^{\mathrm{b}}$ \\
\hline $\mathrm{C} 22: 1, \omega-9$ trans & trans Brassídico & $0,48^{\mathrm{ab}}$ & $0,55^{\mathrm{ab}}$ & $0,37^{\mathrm{b}}$ \\
\hline $\mathrm{C} 24: 1, \omega-9$ cis & Nervônico & $0,35^{\mathrm{a}}$ & $0,15^{\mathrm{bc}}$ & $0,00^{\mathrm{d}}$ \\
\hline \multicolumn{5}{|l|}{ POLINSATURADOS } \\
\hline $\mathrm{C} 18: 2, \omega-6,9 \mathrm{cis}$ & Linoleico & $2,32^{\mathrm{bc}}$ & $2,28^{\mathrm{c}}$ & $1,97^{\mathrm{c}}$ \\
\hline $\mathrm{C} 18: 3, \omega-6,9,12 \mathrm{cis}$ & $\gamma$-linolênico & $0,11^{\mathrm{ab}}$ & $0,04^{\mathrm{cd}}$ & $0,13^{\mathrm{a}}$ \\
\hline $\mathrm{C} 18: 3, \omega-3,6,9 \mathrm{cis}$ & $\alpha$-linolênico & $0,63^{\mathrm{ab}}$ & $0,51^{\mathrm{cd}}$ & $0,71^{\mathrm{a}}$ \\
\hline $\mathrm{C} 20: 3, \omega-6,9,12 \mathrm{cis}$ & di-homo- $\alpha$-linolênico & $0,01^{\mathrm{c}}$ & $0,00^{\mathrm{c}}$ & $0,00^{\mathrm{c}}$ \\
\hline
\end{tabular}

*letras iguais na mesma linha indicam que não existe diferença significativa ao nível de $1 \%$ de significância ( $p>0,01) . \mathrm{LC}=$ leite caprino, $\mathrm{LM}=$ leite misto e $\mathrm{LV}=$ leite de vaca. 
Tabela 2 - Ácidos graxos presentes nos queijos "Chevrotin" simbiotico com leite de cabra, bovino e misto (\%)

\begin{tabular}{|c|c|c|c|c|c|c|c|c|c|}
\hline $\mathrm{AG}$ & QC2 & QC5 & QCT & QM2 & QM5 & QMT & QV2 & QV5 & QVT \\
\hline $\mathrm{C} 4: 0$ & $0,00^{\mathrm{c}}$ & $0,00^{\mathrm{c}}$ & $0,00^{\mathrm{c}}$ & $0,62^{\mathrm{c}}$ & $4,41^{\mathrm{ab}}$ & $4,21^{\mathrm{ab}}$ & $7,49^{\mathrm{a}}$ & $0,28^{\mathrm{c}}$ & $8,42^{\mathrm{c}}$ \\
\hline C6:0 & $0,10^{\mathrm{d}}$ & $0,05^{\mathrm{d}}$ & $0,04^{\mathrm{d}}$ & $0,21^{\mathrm{d}}$ & $1,59^{\mathrm{ab}}$ & $1.55^{\mathrm{ab}}$ & $1,68^{\mathrm{a}}$ & $0,23^{\mathrm{cd}}$ & $3,04^{\mathrm{d}}$ \\
\hline C8:0 & $0,13^{\mathrm{cd}}$ & $0,15^{\mathrm{cd}}$ & $0,31^{\mathrm{c}}$ & $0,85^{\mathrm{a}}$ & $0,70^{\mathrm{ab}}$ & $0,30^{\text {cd }}$ & $0,80^{\mathrm{a}}$ & $0,22^{\mathrm{cd}}$ & $0,55^{\mathrm{b}}$ \\
\hline C10:0 & $3,67^{b}$ & $3,86^{\mathrm{b}}$ & $2,59^{\mathrm{d}}$ & $4,55^{\mathrm{a}}$ & $2,97^{\mathrm{d}}$ & $1,33^{\mathrm{e}}$ & $2,80^{\mathrm{d}}$ & $1,91^{\mathrm{d}}$ & $1,88^{\mathrm{d}}$ \\
\hline C11:0 & $0,04^{\mathrm{c}}$ & $0,03^{\mathrm{c}}$ & $0,07^{\mathrm{c}}$ & $0,05^{\mathrm{c}}$ & $0,22^{\mathrm{ab}}$ & $0,22^{\mathrm{ab}}$ & $0,29^{\mathrm{a}}$ & $0,07^{\mathrm{c}}$ & $0,37^{\mathrm{a}}$ \\
\hline C12:0 & $2,22^{\mathrm{c}}$ & $2,22^{\mathrm{c}}$ & $1,87^{\mathrm{e}}$ & $2,17^{\mathrm{c}}$ & $2,47^{b}$ & $2,15^{\mathrm{c}}$ & $2,20^{\mathrm{c}}$ & $2,70^{\mathrm{a}}$ & $2,46^{b}$ \\
\hline C14:0 & $7,86^{\mathrm{e}}$ & $7,84^{\mathrm{e}}$ & $6,99^{\mathrm{e}}$ & $7,55^{\mathrm{e}}$ & $8,73^{\mathrm{d}}$ & $9,36^{\mathrm{c}}$ & $8,36^{\mathrm{e}}$ & $10,68^{\mathrm{a}}$ & $9,48^{\mathrm{c}}$ \\
\hline C15:0 & $0,94^{b}$ & $0,89^{\mathrm{c}}$ & $0,78^{\mathrm{e}}$ & $0,89^{\mathrm{c}}$ & $0,84^{\mathrm{c}}$ & $1,06^{\mathrm{a}}$ & $0,91^{\mathrm{b}}$ & $1,04^{\mathrm{a}}$ & $0,90^{\mathrm{c}}$ \\
\hline $\mathrm{C} 16: 0$ & $28,39^{b}$ & $27,80^{\mathrm{b}}$ & $23,83^{\mathrm{e}}$ & $26,93^{\mathrm{d}}$ & $26,77^{\mathrm{d}}$ & $27,65^{\mathrm{d}}$ & $25,87^{\mathrm{d}}$ & $29,30^{\mathrm{a}}$ & $25,68^{\mathrm{d}}$ \\
\hline $\mathrm{C} 17: 0$ & $0,80^{\mathrm{a}}$ & $0,77^{\mathrm{b}}$ & $0,65^{\mathrm{b}}$ & $0,77^{b}$ & $0,66^{\mathrm{c}}$ & $0,67^{\mathrm{c}}$ & $0,68^{c}$ & $0,62^{\mathrm{c}}$ & $0,55^{\mathrm{e}}$ \\
\hline C18:0 & $16,7^{\mathrm{ab}}$ & $16,8^{\mathrm{ab}}$ & $14,55^{\mathrm{c}}$ & $16,9^{\mathrm{ab}}$ & $16,11^{b}$ & $17,46^{\mathrm{b}}$ & $16,10^{\mathrm{b}}$ & $17,60^{\mathrm{a}}$ & $15,10^{\mathrm{b}}$ \\
\hline C19:0 & $0,18^{\mathrm{d}}$ & $0,18^{\mathrm{d}}$ & $0,14^{\mathrm{de}}$ & $0,18^{\mathrm{d}}$ & $0,27^{\mathrm{b}}$ & $0,30^{\mathrm{a}}$ & $0,21^{\mathrm{cd}}$ & $0,29^{\mathrm{b}}$ & $0,25^{\mathrm{cd}}$ \\
\hline C20:0 & $0,28^{\mathrm{bc}}$ & $0,26^{\mathrm{bc}}$ & $0,59^{\mathrm{a}}$ & $0,26^{\mathrm{bc}}$ & $0,24^{b c}$ & $0,26^{\mathrm{bc}}$ & $0,23^{b c}$ & $0,24^{\mathrm{bc}}$ & $0,21^{b c}$ \\
\hline $\mathrm{C} 21: 0$ & $0,36^{\mathrm{b}}$ & $0,36^{\mathrm{b}}$ & $0,44^{\mathrm{a}}$ & $0,28^{\mathrm{bc}}$ & $0,00^{\mathrm{d}}$ & $0,37^{\mathrm{bc}}$ & $0,21^{b c}$ & $0,24^{\mathrm{bc}}$ & $0,28^{b c}$ \\
\hline $\mathrm{C} 22: 0$ & $0,01^{\mathrm{cd}}$ & $0,00^{\mathrm{e}}$ & $3,43^{\mathrm{a}}$ & $0,06^{\mathrm{cd}}$ & $0,08^{\mathrm{cd}}$ & $0,11^{\mathrm{bc}}$ & $0,08^{\mathrm{bc}}$ & $0,11^{b c}$ & $0,08^{\mathrm{e}}$ \\
\hline $\mathrm{C} 23: 0$ & $3,30^{\mathrm{a}}$ & $0,78^{\mathrm{e}}$ & $0,00^{\mathrm{d}}$ & $0,31^{\mathrm{e}}$ & $2,45^{\mathrm{b}}$ & $2,49^{b}$ & $2,62^{b}$ & $1,76^{\mathrm{e}}$ & $1,62^{\mathrm{e}}$ \\
\hline $\mathrm{C} 24: 0$ & $0,27^{\mathrm{a}}$ & $0,24^{\mathrm{bc}}$ & $0,27^{\mathrm{a}}$ & $0,09^{c}$ & $0,20^{\mathrm{b}}$ & $0,20^{\mathrm{b}}$ & $0,15^{\mathrm{b}}$ & $0,15^{\mathrm{b}}$ & $0,08^{\mathrm{c}}$ \\
\hline $\mathrm{C} 14: 1, \omega-5$ cis & $0,31^{\mathrm{d}}$ & $0,29^{d}$ & $0,24^{\mathrm{d}}$ & $0,28^{d}$ & $0,37^{\mathrm{b}}$ & $0,95^{\mathrm{a}}$ & $0,37^{\mathrm{d}}$ & $0,69^{\mathrm{bc}}$ & $0,74^{\text {bc }}$ \\
\hline $\mathrm{C} 15: 1, \omega-5$ cis & $0,30^{\text {cd }}$ & $0,29^{\mathrm{cd}}$ & $0,25^{\mathrm{cd}}$ & $0,30^{\text {cd }}$ & $0,27^{\mathrm{cd}}$ & $0,39^{\mathrm{ab}}$ & $0,33^{\mathrm{cd}}$ & $0,36^{\mathrm{ab}}$ & $0,32^{\text {cd }}$ \\
\hline $\mathrm{C} 16: 1, \omega-7$ cis & $0,75^{\mathrm{cd}}$ & $0,81^{\mathrm{cd}}$ & $0,54^{\mathrm{d}}$ & $0,79^{\text {cd }}$ & $0,87^{\mathrm{cd}}$ & $1,31^{\mathrm{ab}}$ & $0,86^{\mathrm{cd}}$ & $1,18^{\mathrm{bc}}$ & $1,11^{\text {cd }}$ \\
\hline $\mathrm{C} 17: 1, \omega-7$ cis & $0,25^{\mathrm{a}}$ & $0,26^{\mathrm{a}}$ & $0,18^{\mathrm{b}}$ & $0,27^{\mathrm{a}}$ & $0,20^{\mathrm{b}}$ & $0,20^{\mathrm{b}}$ & $0,20^{\mathrm{b}}$ & $0,18^{\mathrm{b}}$ & $0,16^{\mathrm{b}}$ \\
\hline C18:1, $\omega-9$ trans & $26,7^{\mathrm{ab}}$ & $29,15^{\mathrm{a}}$ & $19,8^{\mathrm{cd}}$ & $29,02^{\mathrm{a}}$ & $22,8^{\mathrm{cd}}$ & $26,1^{\mathrm{bc}}$ & $22,1^{\mathrm{cd}}$ & $23,5^{\mathrm{bc}}$ & $21,7^{\mathrm{cd}}$ \\
\hline $\mathrm{C} 18: 1, \omega-11 \mathrm{cis}$ & $2,31^{\mathrm{cd}}$ & $2,49^{\mathrm{cd}}$ & $1,75^{\mathrm{d}}$ & $2,55^{\mathrm{cd}}$ & $2,33^{\mathrm{cd}}$ & $3,17^{\mathrm{ab}}$ & $2,47^{\mathrm{cd}}$ & $3,00^{\mathrm{ab}}$ & $2,60^{\mathrm{d}}$ \\
\hline $\mathrm{C} 20: 1, \omega-9$ cis & $0,12^{\mathrm{bc}}$ & $0,30^{\mathrm{a}}$ & $0,30^{\mathrm{a}}$ & $0,42^{\mathrm{a}}$ & $0,36^{\mathrm{a}}$ & $0,12^{\mathrm{bc}}$ & $0,00 \mathrm{~d}$ & $0,43^{\mathrm{a}}$ & $0,11^{\mathrm{bc}}$ \\
\hline C22:1, $\omega-9$ trans & $0,67^{\mathrm{a}}$ & $0,44^{\mathrm{bc}}$ & $0,14^{\mathrm{bc}}$ & $0,23^{\mathrm{bc}}$ & $0,49^{\mathrm{bc}}$ & $0,47^{\mathrm{bc}}$ & $0,36^{\mathrm{bc}}$ & $0,41^{\mathrm{bc}}$ & $0,21^{b c}$ \\
\hline $\mathrm{C} 24: 1, \omega-9 \mathrm{cis}$ & $0,28^{\mathrm{ab}}$ & $0,09^{\mathrm{cd}}$ & $0,35^{\mathrm{a}}$ & $0,03^{d}$ & $0,27^{\mathrm{ab}}$ & $0,33^{\mathrm{a}}$ & $0,23^{\mathrm{ab}}$ & $0,15^{\mathrm{cd}}$ & $0,09^{\mathrm{cd}}$ \\
\hline $\mathrm{C} 18: 2, \omega-6,9 \mathrm{cis}$ & $2,63^{\mathrm{ab}}$ & $3,13^{\mathrm{a}}$ & $2,03^{c}$ & $2,97^{\mathrm{a}}$ & $2,92^{\mathrm{a}}$ & $2,33^{\mathrm{c}}$ & $1,91^{\mathrm{c}}$ & $2,34^{\mathrm{c}}$ & $1,65^{\mathrm{c}}$ \\
\hline $\mathrm{C} 18: 3, \omega-6,9,12$ cis & $0,06^{\mathrm{d}}$ & $0,06^{\mathrm{d}}$ & $0,02^{\mathrm{d}}$ & $0,10^{\text {cd }}$ & $0,08^{\mathrm{cd}}$ & $0,12^{\mathrm{ab}}$ & $0,05^{\mathrm{d}}$ & $0,11^{\mathrm{ab}}$ & $0,07^{\mathrm{d}}$ \\
\hline $\mathrm{C} 18: 3, \omega-3,6,9 \mathrm{cis}$ & $0,33^{\text {cd }}$ & $0,64^{\mathrm{ab}}$ & $0,23^{\mathrm{d}}$ & $0,68^{\mathrm{ab}}$ & $0,33^{\text {cd }}$ & $0,63^{\mathrm{ab}}$ & $0,38^{\mathrm{cd}}$ & $0,50^{\mathrm{bc}}$ & $0,51^{\mathrm{bc}}$ \\
\hline $\mathrm{C} 20: 3, \omega-6,9,12$ cis & $0,00^{\mathrm{c}}$ & $0,00^{\mathrm{c}}$ & $0,06^{\mathrm{b}}$ & $0,04^{\mathrm{b}}$ & $0,27^{\mathrm{a}}$ & $0,00^{\mathrm{c}}$ & $0,00^{\mathrm{c}}$ & $0,00^{\mathrm{c}}$ & $0,00^{\mathrm{c}}$ \\
\hline
\end{tabular}

*letras iguais na mesma linha indicam que não existe diferença significativa ao nível de $1 \%$ de significância $(\mathrm{p}>0,01) . \mathrm{QC} 2=$ queijo de cabra $2 \%$ de inulina, QC5 = queijo de cabra $5 \%$ de inulina, $\mathrm{QCT}=$ queijo de cabra $\mathrm{O} \%$ de inulina, $\mathrm{QM} 2=$ queijo misto $2 \%$ de inulina, QM5 = queijo de misto $5 \%$ de inulina, $\mathrm{QMT}=$ queijo de misto O\% de inulina, QV2 = queijo de vaca $2 \%$ de inulina, QV5 = queijo de vaca 5\% de inulina, $\mathrm{QVT}=$ queijo de vaca $\mathrm{O} \%$ de inulina. 
Comparando o leite bovino, caprino e misto, observou-se uma diferença estatística na maioria dos resultados, ocorrendo uma prevalência dos ácidos fisioterio (C14:1), palmítico (C16:0), palmitoléico (C16:1) e elaidico (C18:1n9C) no leite bovino e dos ácidos palmítico (C16:0), margárico (C17:0), elaídico (C18:1n9T) e linoleico no leite caprino, sendo que o ácido palmítico não demonstrou diferença entre o leite bovino e caprino.

De acordo com os resultados obtidos, os percentuais de ácidos graxos saturados (AGS), a concentração dos ácidos monoinsaturados (MUFA) e a concentração dos ácidos poliinsaturados (PUFA) aumentaram linearmente $(\mathrm{p}<0,01)$. Foram identificados trinta ácidos graxos, dentre estes 17 saturados, nove monossaturados e quatro polinsaturado. Em relação à composição dos ácidos graxos, o leite misto apresentou maior teor de ácidos graxos saturados e poli-insaturados.

Os teores de ácidos graxos dos leites variaram intensamente, divergindo de forma significativa, pois diversas variáveis (espécie, raça, processamento, obtenção da amostra, dieta dos animais, entre outras) devem ser levadas em consideração na caracterização cromatográfica do leite, segundo Chilliard e Ferly (2004); Sanz Sampelayo et al. (2007). O perfil de ácidos graxos das amostras de queijo "Chevrotin" com diferentes tipos de leite e diferentes níveis de inulina, está listados conforme mostra a Tabela 2 .

$\mathrm{O}$ tratamento QV2 apresentou maiores concentrações dos ácidos graxos C:4, C:6, $\mathrm{C}: 8$ e $\mathrm{C}: 11$, sendo significativamente superior aos demais $(\mathrm{p} \leq 0,01)$. Já o tratamento QM5 apresentou maiores concentrações dos áci-dos graxos C:4, C:6, C:8, C:11, C:12, $\mathrm{C}: 14, \mathrm{C}: 15$ e $\mathrm{C}: 16$, sendo significativamente $(\mathrm{p} \leq 0,01)$. Para o tratamento QMT o ácido graxo que teve menor concentrações foi o C:4, e para o tratamento QM2 a menor concentração foram dos ácidos graxos C:8 e
C:10 e para o tratamento QC2 foram os ácidos graxos C:17, C:23 e C:24.

Para o tratamento QCT os ácidos graxos em maiores concentrações foram $\mathrm{C}: 18$, C:20, C:21 e C:22, sendo significativamente superior aos demais $(\mathrm{p} \leq 0,01)$. As maiores concentrações encontradas de ácidos graxos monoinsaturados ocorreram principalmente nos queijos de leite caprino e misto com $2,5 \%$ e $5 \%$ de inulina, sendo significativamente superior aos demais queijos $(\mathrm{p} \leq 0,01)$. Apenas o ácido graxo C18: 1n11 apresentou maior concentração no queijo bovino QV2. Os ácidos graxos poliinsaturados apresentaram maior concentração, principalmente nos queijos de leite caprino e misto com $2,5 \%$ e $5 \%$ de inulina, sendo superior aos demais tratamentos $(\mathrm{p} \leq 0,01)$.

\section{CONCLUSÕES}

A utilização da adição do prebiótico (inulina) e do probiótico (Bifidobacterium lactis) e identificação do perfil de ácidos graxos no queijo tipo Chevrotin de leite bovino, caprino e misto com, através da cromatografia resultou na identificação de trinta ácidos graxos, dentre estes foram dezessete saturados, nove monoinsaturados e quatro poliinsaturados. A composição dos ácidos graxos nos diferentes tipos de queijos apresentou maior teor de ácidos graxos monoinsaturados e poliinsaturados nos queijos caprinos e misto e maior teor de ácidos graxos saturados nos queijos com leite bovino.

\section{REFERÊNCIAS}

AGÊNCIA NACIONAL DE VIGILÂNCIA SANITÁRIA (ANVISA). Resolução RDC $\mathrm{n}^{\mathrm{o}} 2$, de 07 de janeiro de 2002. Aprova o Regulamento Técnico de Substâncias Bioativas e probióticos isolados com alegação de Propriedade funcional e ou de Saúde. Diário Oficial da República Federativa do Brasil, Brasília, 9 jan. 2002. 
BIELECKA, M. Selection of probiotic and prebiotics for synbiotcs and confirmation of their in vivo effetiveness. Food Research International. v. 35, n. 2/3, p. 125-131, 2002.

CANDIDO, L. M. B.; CAMPUS, A. M. Alimentos funcionais. Uma revisão. Boletim da SBCTA, v. 29, n. 2, p. 193-203, 2005.

CARVALHO, M. P. Manipulando a composição do leite: gordura. $1^{\circ}$ Curso online sobre a qualidade do leite. Milkpoint. 2000.

CHILLIARD, Y.; FERLAY, A. Dietary lipids and forages interaction on cow and goat milk fatty acid composition and sensory properties. Reproduction, Nutrition and Development, v. 44, p. 467-492, 2004.

COSTA, E. N. Influência do Tratamento Térmico sobre Os Ácidos Graxos do Leite Bovino. 2011. 125p. Dissertação apresentada à Universidade Estadual do Sudoeste da Bahia, Itapetinga, BA. 2011.

FOLCH, J. M. A simple method for the isolation and purification on total lipids fron animal tissues. The Journal Of Biological Chemistry Baltimore, v. 226, n. 1, p. 497509, 1956.

HARTMAN, L.; LAGO, B. C. A rapid preparation of fatty methyl esters from lipids. Laboratory Pratice, v. 22, p. 475-477, 1973.

YUHARA, T. T. et al. Produção de Queijo tipo Quark funcional contendo exopolissacarídeos. Revista do Instituto de Laticínios Cândido Tostes, v. 69, n. 6, p. 387-394, 2014.

MILINSK, M. C. et al. Comparative analysis of eight esterification methods in the quantitative determination of vegetable oil fatty acid methyl esters (FAME). Journal of Brazilian Chemical Society, v. 19, n. 8, p. 14751483, 2008.

PRANDINI, A. Different level of conjugated linoleic acid (CLA) in dairy products from Italy. Journal of Food Composition and Analysis, v. 20, n. 6, p. 472-479, 2007.

PELLEGRINI L. G. Análise do perfil de ácidos graxos do leite bovino, caprino e ovino. Synergismus scyentifica UTFPR, v. 07, n. 6, 2014.

ROBERFROID, M. B. Functional Foods Concepts: The case of inulin and oligofructose. Journal of Nutrition, v. 129, n. 7, p. 1398S-1401S, 1999.

SANZ SAMPELAYO, M. R. Influence of type of diet on the fat constituents of goat and sheep milk. Small Ruminant Research, v. 68, p. $42-63,2007$.

SAS. Statistical Analysis System (525). Usei Guid. Cary: SAS Institute. 2014.

SIMIONATO J. I. Composição Química e Quantificação de ácidos graxos com ênfase ao ácido linoleico conjugado (CLA) em leite e derivados. MARINGÁ, 2008.

TVRZICKÁ, E. et al. Analysis of fatty acids in plasma lipoproteins by gas chromatographyflame ionization detection: Quantitative aspects. Analysis Chimica Acta, v. 465, p. 337-350. 2002.

ZIEMER, C.; GIBSON, G. An overview of probiotics, prebiotics and symbiotics in the functional concept: perspectives and future strategies. International Dairy Journal, v. 8, n. 5-6, p. 473-479, 1998. 